\title{
What Is Quantum Information Retrieval?
}

\author{
C.J. Keith van Rijsbergen \\ keith@dcs.gla.ac.uk
}

\begin{abstract}
I will introduce the theoretical foundations for quantum information retrieval derived from Quantum Theory.

There will be an explanation of how such a theoretical framework could be useful in IR, for example, by showing how logic, probability, and geometry, as exploited in IR, can be represented in a consistent way in the underlying Hilbert Space.

The talk will conclude with some examples of recent concrete applications of the framework in IR.
\end{abstract}

